\section{Extremely low birth weight is linked to risk of chronic illness}

Susan Mayor London

Babies with an extremely low birth weight $(<1000 \mathrm{~g})$ have a higher risk of developing chronic health conditions, a new study shows. And as well as a having a higher risk of illnesses such as asthma, these babies are more likely than babies of normal birth weight to have functional and educational limitations, the follow-up study shows (JAMA 2005;294:318-25).

The US study looked at health outcomes at the age of eight years in a group of children who had an extremely low birth weight. The children were 219 survivors of the cohort of 344 extremely low birthweight children admitted to the neonatal intensive care unit at one hospital, Rainbow Babies and Children's Hospital, in Cleveland, Ohio, between 1992 and 1995. They were compared with 176 normal birthweight controls of similar sociodemographic status.

The researchers found that extremely low birthweight children were more than three times as likely as normal birthweight children to have chronic conditions, including functional limitations (64\% v 20\%; odds ratio 8.1 (95\% confidence interval 5.0 to 13.1), after data were adjusted for sociodemographic status and sex.

They were twice as likely to need medical interventions or services to compensate for limited function $(48 \%$ v 23\%; odds ratio 3.0 (1.9 to 4.7$)$ ) and to need more services than those routinely required by children (65\% v 27\%; odds ratio 5.4 (3.4 to 8.5)). These differences remained significant even when the 36 extremely low birthweight children with neurosensory impairments were excluded from the analysis.

Differences for specific diagnoses and disabilities included cerebral palsy $(14 \%$ v $0 \%$; $\mathrm{P}<0.001)$, asthma $(21 \%$ v $9 \%$; odds ratio 3.0 (1.6 to 5.6)), and vision of less than $20 / 200(10 \% v$ $3 \%$; odds ratio 3.1 (1.2 to 7.8$)$ ). Educational deficits included IQ $<85$ (38\% v 14\%; odds ratio 4.5 (2.7 to 7.7)) and limited academic skills $(37 \%$ v $15 \%$; odds ratio 4.2 (2.5 to 7.3)). Poor motor skills (47\% v $10 \%$; odds ratio 7.8 (4.5 to 13.6)) and poor adaptive functioning $(69 \%$ v 34\%; odds ratio 6.5 (4.0 to 10.6)) were also common among the low birthweight group.

Advances in perinatal care in the 1990s-including surfactant therapy and more use of antenatal steroids-have resulted in dramatic increases in the survival of extremely low birthweight infants, according to background information in the article. However, little has previously been known about how these children function at school age.

Maureen Hack, professor of paediatrics and director of high risk follow up at Rainbow Babies and Children's Hospital and lead investigator of the study, said: "Our results reveal that extremely low birthweight children have very high rates of chronic conditions compared with children born at normal weight. These conditions include asthma, cerebral palsy, and visu-

al disability, as well as poorer cognitive ability, academic achievement, motor skills, and social adaptive functioning."

Professor Hack added: "The clinical implications are that the children need comprehensive follow-up and anticipatory guidance and care. Proactive planning for their long term health and educational care needs is essential to optimally treat and possibly improve outcomes through preventative and early intervention services."

She pointed out that although extremely low birthweight children constitute less than $1 \%$ of babies and thus the societal impact is not enormous, the effect on families is enormous.

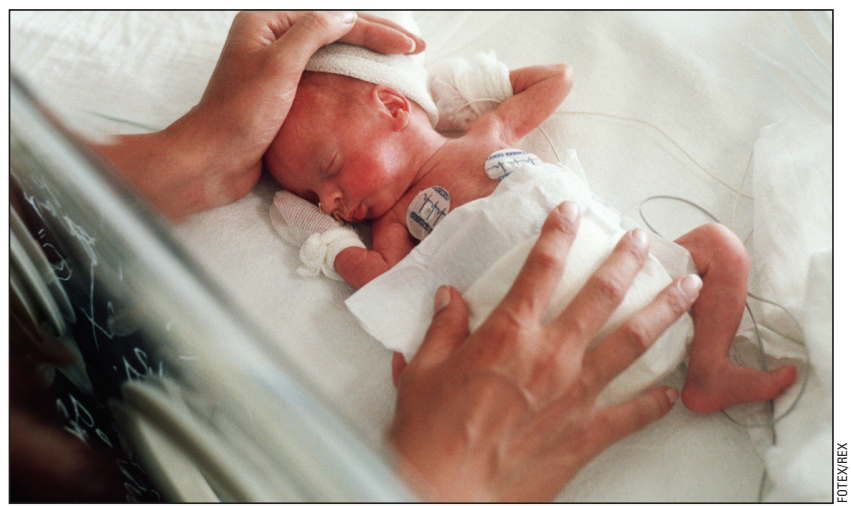

Extremely low birthweight children, like the baby pictured above, are more than three times as likely as normal birthweight children to have chronic conditions, including functional limitations

\title{
Inquiry into high mortality of GPs' patients finds no wrongdoing
}

Roger Dobson Abergavenny

An investigation into five GPs whose patients showed a high mortality has found no evidence of negligence or malpractice.

The review was carried out after the Shipman inquiry was notified of 12 GPs whose patients had an excessively high mortality. The inquiry recommended that the practices be investigated. An inquiry into two of them in the West Midlands, completed last year, had also found no evidence of wrongdoing (BMJ 2004;328: 1474).

The latest investigation found that the large number of nursing home patients on the GPs' lists was likely to have been responsible for the high mortality.

An article published online ahead of print on 6 July in the Journal of Public Health (http:// jpubhealth.oxfordjournals.org, doi: 10.1093/pubmed/fdi043) warns that such inquiries are costly and time consuming.

"The introduction of a national mortality monitoring system for GPs requires careful consideration of the consequences, not only for individual practitioners flagged up as having excessive mortality rates, but also for the primary care organisations that may be expected to conduct follow-up investigations, but in many cases will lack the resources as well as the expertise to carry these out," wrote the authors, from Imperial College, London, the National Clinical Assessment
Authority, and the Adur, Arun and Worthing Primary Care Trust.

"The review enabled us to assess the quality of care provided by these five GPs, and increased our confidence in the final conclusion that there was no evidence of malpractice or negligence. Yet our investigation was resource-intensive, with direct and opportunity costs that ran into several thousands of pounds," they wrote.

The report says that in December 2004 the Shipman inquiry recommended the development of a national system for routinely monitoring death rates among patients of general practices. "Yet it remains unclear who should be responsible for investigating GPs flagged up as having unusually high death rates by such a system, or what methods should be used," it says.

The review involved a retrospective analysis of routine mortality data and patient registration data from five general practices in West Sussex and a review of the case notes of deceased patients. Outcome measures included standardised mortality ratios, the proportion of deaths in nursing homes, and reviewers' concordance with GPs' decisions on issue a death certificate.

Standardised mortality ratios for the practices ranged from 145 to 239 (against a standard set for West Sussex of 100), and all differences from the West Sussex average were statistically significant $(\mathrm{P}<0.02)$.

There was no association between the high death rates and the age and sex composition of each GP's patient population. But the death rates did correlate highly with the proportion of deaths occurring in nursing homes. The results show that more than a third of the deaths occurred in nursing homes (nationally about 11\% of deaths occur in nursing homes). 